\title{
Foundation for behaviour management: A systems approach
}

\author{
Diane Andrews and Trevor Clarke \\ Behaviour Support Team, Group Special Education, Southland
}

\begin{abstract}
This article outlines the development and implementation of a project designed to help a school reflect on how well it has developed supportive behaviour management systems. The programme further encourages the school to implement more functional systems. When the local Group Special Education (GSE) office was offered an opportunity to develop some innovation projects, a proposal for looking at behaviour management as a school wide systems intervention was developed. The conceptual base was to focus on six targeted school systems believed to be the foundations for functional behaviour management, profile them for a school, report them in a way that has meaning and work with the school in improving functionality. The project has been used in seven schools in Southland and one in Canterbury. All the schools reported positive outcomes, and some schools made major advances in developing functional behaviour management systems.
\end{abstract}

\section{KEYWORDS:}

Behaviour management, social culture, school behaviour, whole-school approach, school based intervention.

\section{INTRODUCTION}

In this project, the writers defined the factors that facilitate, or hinder, the development of functional behaviour management systems. When these factors, or systems, were defined it was then necessary to develop ways of measuring them in the field, analysing them and presenting them back to the school in a way that was constructive and had meaning.

In our work as caseworkers in the GSE behaviour support team, we often observe schools and teachers in various stages of developing and implementing behaviour management strategies and policies. With less developed systems there are often few choices left for the poorly behaving student, the school has difficulty focusing resources on students with challenging behaviours and building a support team can be problematical. Schools have often stopped owning the "behaviour problem" and are looking to outside assistance to remove the student.

There are, however, very good examples of positive and functional behaviour management systems. In these schools supporting behaviour has clear pathways and accountabilities, focused resources, and more options. Agencies work together, parents are more available and supportive, and positive gains are acknowledged and built on. There is a support team placed around the student and each member has defined tasks. Much of the support is proactive, providing positive environments in the classroom and playground, and meeting the individual needs of students.

It is not uncommon for schools to search for specific programmes such as Assertive Discipline (Canter, 1992), Teacher Effective Training (Gordon, 1974), or Positive Behaviour Support (Sugai, 1994), that will address behaviour issues and, in doing so, fail to recognise that the successful management of behaviour encompasses a range of systems which all contribute to success. These packages may well encourage good class tone and provide a structure for teachers, but unless they are embedded in school systems, which reflect the desired culture of the school, it is likely that they will be operating in isolation. The programmes may give the school a sense of security, but are seldom responsive to specific school communities and sometimes lack the flexibility to respond to individual student needs.

The implementation of a one-off behaviour management system in a school can lead to the expectation that this will be a solution to all discipline "problems". Behaviour management cannot be an "add-on" but needs to be an integral part of selected school systems.

\section{CONCEPTUAL BASIS}

The potential of school-wide interventions have long been recognised. Eaden (2004) notes a number of reports (Eber, 2002; Gill, 1989; Knoster, 2002; Nelson, 1998; Shaunessy, 2002) that advocate the "immense contribution" school-wide systems of behavioural intervention can make. Some students with behavioural and emotional difficulties do not fare well in regular schools and require school wide systems to enable "wraparound approaches" with individual plans and positive environments. Eaden (2004) reports White (2001) as defining unified expectations based around consistency, clear expectations, roles and responsibilities. This is a team or whole school responsibility and not the domain of individual teachers only. Shaunessy (2002) advocates a coordinated school-wide system that facilitates early identification and intervention. This increases the possibilities of more successful outcomes.

This project places the responsibility for developing positive school organisational change not on any single individual but with all the individual and collective components of the school. The school organisation is a collection of these systems. Managing behaviour has become increasingly 
important to the organisational pattern of a school. The effectiveness of behavioural management strategies and programmes will be dependent on the effectiveness of the school organisation as a whole.

The writers determined six "foundation systems" for behaviour management through reviewing their own practice, reflecting on their own teaching experience, looking at accumulated data over several years of behaviour support work, researching existing approaches and interventions being used within GSE, and by reviewing current schoolbased programmes. Six interrelated systems within the school are targeted - pastoral care, valuing diversity, discipline, effective teaching, professional development and leadership. A review of the development and function of each of these systems produced a profile of the foundations necessary for supporting an effective behaviour management system.

The discipline system, which is sometimes the sole focus for behaviour management, is only part of the whole behaviour management structure in the school. In the profile it had equal emphasis with the other five systems. An effective discipline system will be positive and school-wide but still offer flexibility and responsibility.

The profile reported not on the standard of teaching in the school, but rather the systems that are in place to ensure that there is effective teaching. Some of these will be based around appraisal, shared planning, understanding of behaviour and the promotion of positive environments.

A system that makes certain a school values diversity will acknowledge the micro cultures of the school and community and ensure that programmes are in place to support these. It is important that individuals and groups are valued and are part of the whole school community.

The leadership system in a school relies on excellent communication and collective and individual responsibility. It needs to be inclusive in practice. Although it is important that leadership begins at the top, all individuals can have some leadership role.

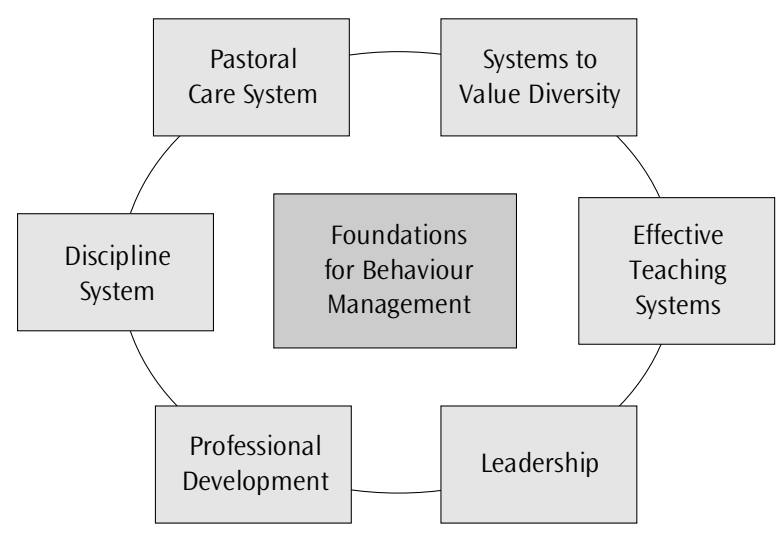

The professional development system is an ongoing process enabling the school to make change and shape its school culture. It needs to be adequately resourced and supported. It can be an investment in both individual and whole school developments.

The need for a formal pastoral care system to proactively identify and plan for the needs of students and staff is an essential component of a behaviour management system. It creates a positive environment with structured supports for the school community.

These systems, when fully functional, will have evidence of the following qualities: -positive environments, inclusive practices, collective and individual ownership, consultative communication, and nonaversive strategies. These qualities help produce positive outcomes, commitment, partnership, positively shaped systems and longer lasting change.

\section{THE PROJECT STORY}

In 2001 the Southland Special Education office applied for surplus national money that was available for special projects. With this, the Behaviour Support Team seconded two full-time equivalent staff for one term to allow individuals time to pursue proposed innovation projects. The requirement was that each project should result in a permanent product that would contribute to our work in schools. A project focusing on looking at the systems underpinning good behaviour management was established.

This idea was presented to a local reference group made up of the Special Education District Manager, a local Principal and a Resource Teacher of Learning and Behaviour (RTLB). Feedback from this group indicated that the concepts on which the programme was based were acceptable and it was likely that the results of a profile would enable a review of behaviour management in a school and provide data to reflect on current systems.

The development of specific tools that would collect accurate data were trialled and retrialled at a local school. Separate and specific questionnaires were designed to gather information on each of the six systems.

A formal school-wide trial was proposed. A school was identified and the Principal was approached with a request to trial the programme in their school. There was a clear understanding that the trial would provide an opportunity for the school to review its behaviour management systems and provide feedback to the authors regarding both the process and the outcome of the profile.

The authors spent two and a half days in the school. During this time, 45-minute interviews were completed with each teacher. All support staff, including the office person and caretaker, and a random selection of students were interviewed for about 15 minutes each. Some parents, Board of Trustee (BOT) members and the local RTLB were interviewed after school or in the evening. Observations were undertaken in each classroom and in the grounds at break 
times using specifically designed observation sheets. The Principal was asked to produce documentation such as relevant policies, guidelines and newsletters from a list provided. This was not reviewed at the time but taken back to the office to add to the data gathered to be used during the report writing phase.

As a result of this initial trial some changes were made to individual questionnaires. The interview schedules for BOTs, parents and outside agencies were reshaped so they could be conducted by telephone. This enabled greater flexibility to ensure sufficient contacts were made, and it was found that the quality of this data was just as informative. The list of possible outside agencies that could be contacted was extended to include such personnel as Health Nurses, Resource Teachers of Reading, Resource Teachers of Māori and School Chaplains.

The preparation of the report required considerable triangulation of data. It was a compilation of accurate information and does not judge or condemn. The narrative was reduced to about two pages on each system and was concluded by a chart that highlighted the presence of important components of each system. Nothing could be written that did not come from several sources of information. Neither in the trial school or the six subsequent schools has there been any questioning of the accuracy of data.

The report was presented orally at a meeting of all staff and the BOT. This took approximately an hour and a half. A brief overview of the whole project was given, followed by individual system profiles. Extensive use was made of visuals. An initial response to the report was part of the presentation but the school was advised to set a later date for a full discussion. This enables written copies of the report to be studied in further detail and management can design an appropriate strategy for using the report.

Some schools have opted into a "probe" report each year. This can mean a full review of all six systems, but others have focused on specific systems. This requires less practitioner time and uses modified data gathering tools. The work still covers the principles behind each of the systems and may add some more targeted questions related to specific interventions.

Peer review of the programme and the process was an important component in updating and refining the tools. Other Psychologists and Special Education advisers in the local office joined teams undertaking the work in schools. The programme was given brief exposure during the first of the GSE South Island Roadshows and interest was shown from both other practitioners and from individual districts. More recently two workshops have been held in the Canterbury district for practitioners interested in the programme.
Guidelines ensure there is little variance from agreed procedures. In Canterbury, a team of three, after completing a daylong workshop, worked alongside the original practitioners to complete the profile in a school. A local coordinator has now been appointed to organise future teams and schools. The three who have undertaken the work in the first school are now in a position to lead their own teams.

During development, the challenge was to ensure the programme would be applicable in all schools. To date it has been used in contributing schools, full primaries, an area school, two intermediates, a secondary school and an integrated school. The logistics of carrying out a full profile in a large secondary school has yet to addressed but the data can be collected in a school of up to 500 students by using the equivalent of three staff for three days.

\section{OUTCOMES}

For some schools the report has been an affirmation of current practices. For others it highlighted several areas where systems have not existed or been functional. In some cases it clearly defined major areas for development and created a need for comprehensive planning and reorganisation.

Eaden (2004) reported in his review of seven schools undertaking the programme that six had made changes as a direct result of the profile. The most significant areas were in developing systems supporting pastoral care, valuing diversity and effective teaching. Four of the seven schools had actively sought help from outside agencies to address concerns and five of the seven schools reported positive improvement in student behaviour.

Review comments from the schools highlighted the following points:

- the value of having specialised information regarding core areas

- the programme was more effective than an Education Review Office report in bringing about change

- the process was thorough, sensitive and supportive.

Since the programme was implemented, schools have continued to report improved behaviour management and outcomes for individual students, focused planning, more formalised systems, and planned opportunities for reflection and review. They also continue to collect and monitor their own data. Schools have used the profile to report both general and specific information to parents.

Other schools have acknowledged the programme highlighted areas of need and gave them a starting point. In one school the BOT reported the profile helped them see the severity of the situation and gave them the confidence and motivation to make changes. 


\section{DISCUSSION}

Although the project has been developed over a number of years it continues to be modified. We feel a number of points are clear.

1. A profile of functional school systems can be produced and this can be presented constructively and accurately to support a school.

2. Individual casework, and generally the ability to support a school, is significantly enhanced where the profile has taken place.

3. Schools are more likely to engage in professional development or school wide development after receiving the profile.

4. A key factor in changing attitude and school culture is the recognition that there are important inter-related systems which support behaviour management. The profile report highlights these systems and provides a formal structure for focused interventions. The probe report acknowledges and affirms progress, and provides a further opportunity for self review.

5. Pastoral care support and the way a school acknowledges diversity are often the first focus areas in this process.

6. A school needs time to make such changes - the profile exercise needs to be seen as part of a year long intervention at least.

7. Commitment from the principal and BOT, along with appropriate contracting, including negotiation of a service agreement, is an important ingredient for success.

Three issues have arisen for us as developers of the programme. Firstly, it was initially envisaged that prepackaged modules for each of the systems could be delivered to any school that has a defined "weakness" in any particular system. However, the eventual diversity of interventions implemented by schools has been one of the strengths coming out of the profile report. This ability for schools to access specific help to strengthen an individual system has both encouraged ownership by the schools and ensured that the most appropriate help is provided. In this way the profile is complementary to other programmes.

Secondly, the question of whether the profile is an appropriate tool for a school in crisis has not been completely answered. A school in crisis may find it difficult to accept the presentation of the data collected. There are no interim strategies to provide immediate steps to avert risks. However, the system of gathering data is appropriate and the report can provide excellent baseline data. The need for each of the six systems to be functioning is important in any school. There can be an element of "relief" as existing problems are recorded and laid on the table for full and frank discussion. The programme can provide a structure for targeted interventions, and a more comprehensive focus on behaviour from a systems perspective encourages individual and collective responsibility among staff. The programme was not written to specifically focus on a school in crisis but rather as an opportunity for self-reflection for staff in any school.

Finally, the confidentiality of the information collected and reported is important. Some of this information is sensitive and designed for self-reflection rather than publication. As with other work within GSE, such as Eliminating Violence reports, there are unclear boundaries about how the information can be handled.

The project has enabled us to learn much about working with schools and has been very rewarding. Continuing to build up experience, broaden the base and revise the various tools will be essential. There is much to be gained from having a constructive process when working with schools not only in terms of increasing the likelihood or appropriate behaviour occurring but also to lift school awareness of the interrelationship between the different systems within their organisations, (Eaden, 2004).

\section{REFERENCES}

Canter, L. \& M. (1992). Assertive discipline: Positive behaviour management for today's classroom. Santa Monica, CA: Canter and Associates.

Eaden, G. (2004). Foundations of Behaviour Management. Unpublished report for Masters requirements.

Gordon, T. (1974). Teacher Effectiveness Training. New York: Wyden Books.

Sugai, G. (1996). Providing effective behaviour support to all students: Procedures and processes. SAIL, 11(1), 1-4

\section{PROFILE OF AUTHORS}

\section{Diane Andrews}

Diane Andrews taught for twenty years in a range of primary, intermediate and secondary schools throughout New Zealand. She joined SES (GSE) in 1998 and spent the next six and a half years as part of the behaviour service. A significant focus of her position was a focus on school systems development. Diane is currently teaching in a secondary school.

\section{Trevor Clarke}

Trevor Clarke taught for sixteen years in several areas of New Zealand before joining SES in 1990 and retraining as a psychologist. During his time with SES, and latterly GSE, Trevor has been involved in behaviour support and has a particular interest in developing systems support programmes for schools that increase capability and support individual casework.

\section{ACKNOWLEDGEMENT}

The authors would like to acknowledge the support and ongoing encouragement of our GSE colleagues and, in particular, Murray Witheford, Southland District GSE Manager. 